\title{
Angular momentum of optical modes in a silicon channel waveguide
}

\author{
Dengke Zhang ๑* $^{*}$ \\ Department of Physics, Tokyo University of Science, Kagurazaka, Tokyo 162-8601, Japan
}

(Received 29 April 2020; accepted 31 August 2020; published 21 September 2020)

\begin{abstract}
The spin and orbital angular momentum (OAM) of optical fields in a silicon channel waveguide are investigated. A vortex beam carrying OAM can be generated by a superposition of two quasi-TE modes. The longitudinal OAM of the superposed vortex beam is intrinsic and mediated by the superposition coefficient. Due to high transverse confinement of the silicon waveguide, the polarization and OAM are inevitably coupled. Their hybridization degree is well described by joint average transverse wave vectors. Further, the OAM part can be separated using the topological Pancharatnam charge and Stokes parameters. Combining polarization and OAM indices, we find that the whole space of structure parameters of the waveguide can be divided into three regimes. Our result offers insight into the relationship between angular momentum and mode confinement, which is beneficial for potential applications of vector beams in photonic integrated circuits.
\end{abstract}

DOI: 10.1103/PhysRevResearch.2.033454

\section{INTRODUCTION}

Light possesses a set of dynamical characteristics of energy, momentum, and angular momentum (AM) [1]. The AM can be separated into spin and orbital contributions due to the vectorial nature of the electromagnetic fields [2,3]. The spin angular momentum (SAM) is related to the intrinsic polarization degrees of freedom. The spatial distribution and propagation of the optical fields determine the orbital angular momentum (OAM). Stemming from the internal coupling properties of Maxwell's equations, the interaction between the polarization and the orbital degrees of freedom happens naturally [4]. This spin-orbit interaction of light has a fundamental importance in optical processes and explains many striking phenomena involving the areas of nano-optics, plasmonics, nonlinear optics, and quantum informatics [5-11].

Over the past decades, the SAM and OAM of light have been well studied for free-space electromagnetic fields, even nonparaxial [12-14]. Recently, these concepts have entered the light-wave guiding systems (e.g., optical fibers) arising from the important application for information processing [15-17]. The guiding systems intimately relate to inhomogeneous optical media, where structured lights are supported. An important category of guiding systems is optical fiber, wherein the angular momentum has been studied by many researchers [17-20]. Most optical fibers are weakly guiding systems and propagation of lights approaches paraxial, resulting in decoupling between the spin and the

\footnotetext{
*dkzhang@outlook.com

Published by the American Physical Society under the terms of the Creative Commons Attribution 4.0 International license. Further distribution of this work must maintain attribution to the author(s) and the published article's title, journal citation, and DOI.
}

orbital degrees of freedom. However, the spin-orbit coupling inevitably appears under the consideration of transverse confinement and removes the independence of SAM and OAM. Now it is well known that the guided light beams propagating in an inhomogeneous media possess mutually coupled SAM and OAM [21-23]. As a result, the polarization and the propagation of optical beams are connected via the spin-orbit interaction of light in the guiding systems. In particular, the concepts of transverse spin and spin-momentum locking in evanescent waves (associated with the guided waves) have been proposed to achieve spin-controlled directional guiding of light [24-26].

More recently, another important guiding system - the silicon waveguide-has attracted great attention due to the pursuits of robustness, miniaturization, and energy efficiency of photonic integrated circuits, where the silicon waveguide is the basic unit and component $[27,28]$. Because of the highly confined modes in silicon waveguides, the SAM and OAM are strongly coupled. The spin-orbit interaction of light in silicon waveguides promises to be a powerful tool available for fabricating integrated optical devices with special functionality such as polarization rotation, directional guiding, chiral sensing, and SAM-to-OAM transformation [29-36]. Due to the planar technology, the silicon channel waveguide is the most common structure and compatible with current technologies of integrated optical circuits. It is important to note that vortex modes carrying OAM are naturally supported in cylindrically symmetric waveguides (e.g., optical fibers). Apparently, the rotation invariant is no longer present in channel waveguides. Therefore, compared with the typical weak-guide fibers, the silicon channel waveguides are strongly confined optical structures without cylindrical symmetry. This distinct difference may bring special properties of angular momentum and hold great promise for studying many intriguing phenomena such as the spin-Hall effect of light, extraordinary optical momentum, spin-orbit hybrid entangled states, and quantumoptical analogies [37-42]. 
In our previous work, we proposed that a first-order OAM field can be generated in a $\mathrm{Si} / \mathrm{SiO}_{2}$ channel waveguide with the superposition method [33]. In that paper, two quasi-transverse electric eigenmodes, $\mathrm{TE}_{01}$ and $\mathrm{TE}_{10}$, were superposed to generate first-order OAM fields. Meanwhile, we designed a coupling structure as an OAM generator with the excitation of fundamental modes and calculated the transformation efficiency (i.e., purity) of the OAM charges by the expansion method. It should be noted that the calculation of the charge purity was based on the common assumption that the charge of the OAM basis (perfect superposition) itself is \pm 1 , which corresponds to the mode azimuthal order (i.e., one node in the transverse field distribution) of $\mathrm{TE}_{01} / \mathrm{TE}_{10}$ (see the Appendix for details). However, note that there is no rotationinvariant symmetry in the silicon channel waveguide and its modes are highly confined due to the large refractive index difference. These conditions are different from free-space and cylindrical waveguides and make the assumption unreliable. In this regard, it is necessary to reexamine the OAM value of generated modes in such a channel waveguide to shed light on the distinctiveness.

In this work, we investigate systematically the angular momentum behaviors of superposed modes of $\mathrm{TE}_{01}$ and $\mathrm{TE}_{10}$ in a $\mathrm{Si} / \mathrm{SiO}_{2}$ channel waveguide. The modulation of the superposition coefficient on OAM and SAM is demonstrated. The hybridization of OAM and SAM, induced by the high transverse confinement, is analyzed in the spatial frequency domain. In particular, we find that the separation of OAM density can be well described by topological Pancharatnam charge and Stokes parameters. This paper is organized as follows. In Sec. II, first we introduce the formalism for angular momenta in inhomogeneous media. Then we demonstrate the basic feature of angular momenta in a silicon channel waveguide. And the topological Pancharatnam charge is depicted for characterizing helical phases. Section III shows the maximum achievable OAM charge in a silicon channel waveguide and unfolds the hybridization of OAM and SAM via three special cases. We develop the average transverse wave vector for the description of confinement and build the correspondence with the hybridization of OAM and SAM. Separation of the OAM density is achieved with the use of topological Pancharatnam charge and Stokes parameters. Finally, we present the conclusion in Sec. IV.

\section{THEORY}

\section{A. Formalism for the angular momentum}

To tackle angular momenta in an inhomogeneous medium, we introduce an effective formalism describing the canonical momentum, spin, and orbital AM of light in isotropic dispersive media, which is developed and examined recently in cylindrical dielectric fibers and metallic wires [19,43]. First, a cycle-integral energy density in such a medium can be described by the Brillouin expression [44]

$$
W=\left(\tilde{\varepsilon}|\mathbf{E}|^{2}+\tilde{\mu}|\mathbf{H}|^{2}\right) / 4,
$$

where $\mathbf{E}(\mathbf{r})$ and $\mathbf{H}(\mathbf{r})$ are the complex electric and magnetic field amplitudes, and $\{\tilde{\varepsilon}, \tilde{\mu}\}=\{\varepsilon, \mu\}+\omega \mathrm{d}\{\varepsilon, \mu\} / \mathrm{d} \omega$ are the dispersion-modified absolute permittivity $\varepsilon(\mathbf{r}, \omega)$ and permeability $\mu(\mathbf{r}, \omega)$, with $\omega$ the angular frequency of light. Then the canonical momentum, spin, and orbital AM densities are expressed as $[19,43]$

$$
\begin{aligned}
\mathbf{P} & =(4 \omega)^{-1} \operatorname{Im}\left[\tilde{\varepsilon} \mathbf{E}^{*} \cdot(\nabla) \mathbf{E}+\tilde{\mu} \mathbf{H}^{*} \cdot(\nabla) \mathbf{H}\right], \\
\mathbf{S} & =(4 \omega)^{-1} \operatorname{Im}\left[\tilde{\varepsilon} \mathbf{E}^{*} \times \mathbf{E}+\tilde{\mu} \mathbf{H}^{*} \times \mathbf{H}\right], \\
\mathbf{L} & =\mathbf{r} \times \mathbf{P} ;
\end{aligned}
$$

here we have used the notation $\mathbf{E}^{*} \cdot(\nabla) \mathbf{E}=\sum_{i} E_{i}^{*} \nabla E_{i}(i=$ $x, y, z)$. As demonstrated in Ref. [19], for cylindrical waveguides, considering a guided mode with propagation constant $\beta$ along the $z$ direction, the longitudinal momentum and energy fulfill the relation $\left\langle P_{z}\right\rangle /\langle W\rangle=\beta / \omega$, where $\langle\ldots\rangle$ denotes the integration over the transverse $(x-y)$ plane.

\section{B. Angular momentum of superposed modes in silicon channel waveguides}

Similarly to the structure parameters of the $\mathrm{Si} / \mathrm{SiO}_{2}$ channel waveguide in Ref. [33], the silicon core with a cross section of $720 \mathrm{~nm} \times 600 \mathrm{~nm}$ is embedded in silicon dioxide. The corresponding refractive indices are set at $n_{\mathrm{Si}}=3.45$ and $n_{\mathrm{SiO}_{2}}=1.46$. For simplicity, only nondispersive and lossless media are considered in what follows, thus $\{\tilde{\varepsilon}, \tilde{\mu}\}=$ $\{\varepsilon, \mu\}$. It can be calculated that $\mathrm{TE}_{01}$ and $\mathrm{TE}_{10}$ are degenerate at wavelength $\lambda=1550 \mathrm{~nm}$ with propagation constant $k_{z}=$ $10.48 \mathrm{rad} / \mu \mathrm{m}$ (see the Appendix for details). As shown in Fig. 1(a), a vortex beam can be generated by the superposition of $\mathrm{TE}_{01}$ and $\mathrm{TE}_{10}$ with a phase shift of $\pi / 2$. Without loss of generality, the generic superposed field can be expressed in a normalized form,

$$
\mathbf{E}^{\mathrm{sp}}=\frac{\left[(1-\alpha) \mathbf{E}^{(01)}+\alpha e^{i \delta \varphi} \mathbf{E}^{(10)}\right]}{\sqrt{2 \alpha^{2}-2 \alpha+1}},
$$

where $\mathbf{E}^{(01)}$ and $\mathbf{E}^{(10)}$ are power-normalized electric fields of $\mathrm{TE}_{01}$ and $\mathrm{TE}_{10}$, respectively, and $\{\alpha, \delta \varphi\}$ denotes the superposition coefficient with the amplitude weight $(0 \leqslant \alpha \leqslant 1)$ and phase difference $(0 \leqslant \delta \varphi<2 \pi)$. Using Eqs. (1) and (2), we calculate average charges of the canonical OAM $\left(\omega\left\langle L_{z}\right\rangle /\langle W\rangle\right)$ and $\operatorname{SAM}\left(\omega\left\langle S_{z}\right\rangle /\langle W\rangle\right)$ in relation to $\{\alpha, \delta \varphi\}$, which are shown in Fig. 1(b). Apparently, the average OAM charge is mediated by $\{\alpha, \delta \varphi\}$, which is analogous to that of the LaguerreGaussian mode $\mathrm{LG}_{01}$ generated by Hermite-Gaussian modes $\mathrm{HG}_{01}$ and $\mathrm{HG}_{10}$ [2]. However, the tunable behavior of the SAM of $\mathbf{E}^{\text {sp }}$ is different from that of $\mathrm{LG}_{01}$ (which is always 0 ). This occurs because there are no pure TE modes in channel waveguides, so different field components will mix and generate elliptical polarization states in the superposed fields [see Fig. 1(a)]. To assess the intrinsic attribute of OAM in a channel waveguide, we perform relevant calculations for maximally twisted $\mathbf{E}^{\mathrm{sp}}$ at $\{\alpha, \delta \varphi\}=\{0.5, \pi / 2\}$, which is the scenario exactly demonstrated in Fig. 1(a). The calculated distribution of the longitudinal OAM density $\left(L_{z}\right)$ on the waveguide cross section ( $x-y$ plane) is displayed in Fig. 1(c). We examine two cases: no displacement shift (origin of $\mathbf{r}_{\perp}$ coincides with beam center) and a shift of $(100 \mathrm{~nm}, 50 \mathrm{~nm})$ for the origin. The calculations give that the cross-section integral $\omega\left\langle L_{z}\right\rangle /\langle W\rangle$ equals 0.87 in both cases, though their density distributions are different. This characteristic indicates that the generated OAM field has an intrinsic longitudinal orbital angular momentum $[25,45]$. The result that the net transverse 


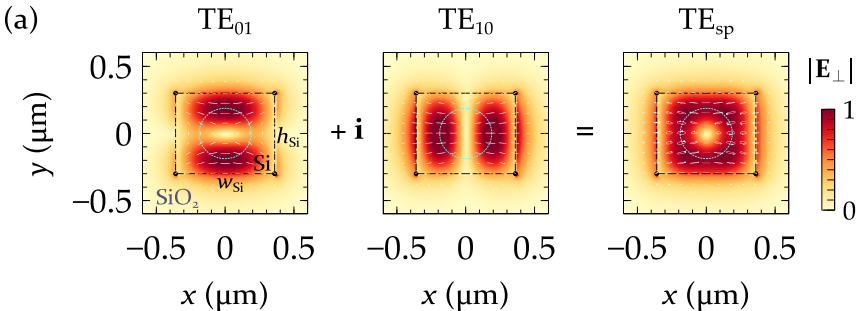

(b)

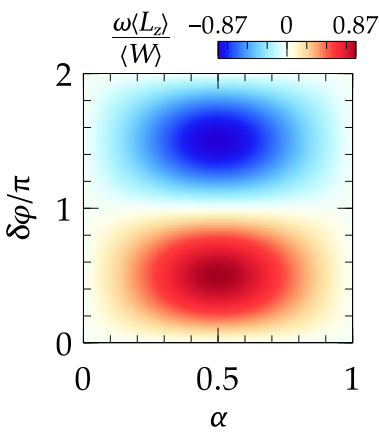

(c)

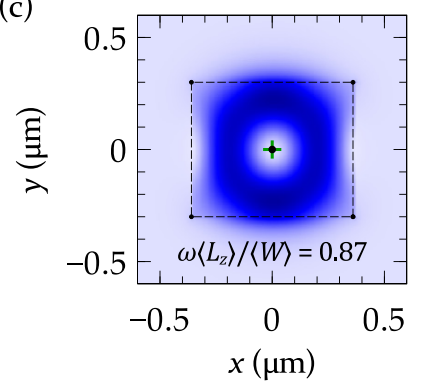

(a)
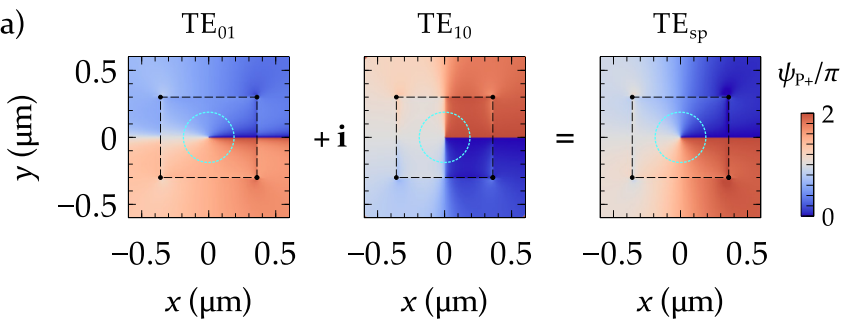

(b)

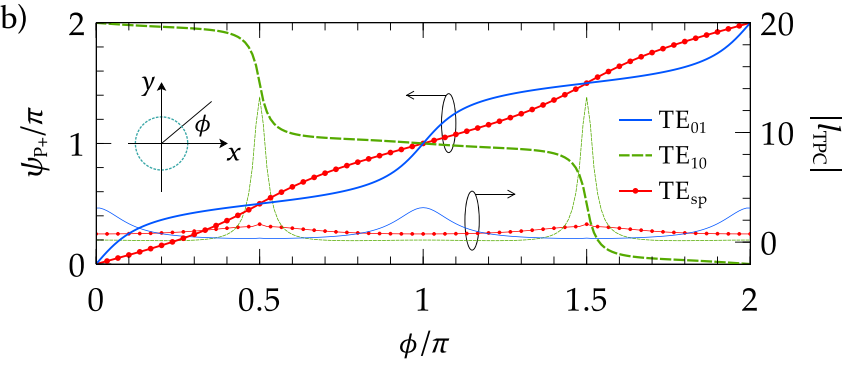

FIG. 2. (a) The phase front $\psi_{\mathrm{P}+}$ based on the Pancharatnam connection with the reference field $\left|e_{+}\right\rangle$for the corresponding fields displayed in Fig. 1(a). The dashed black boxes and dashed cyan circles are identical to those in Fig. 1(a). (b) $\psi_{\mathrm{P}+}$ along the path of the dashed cyan circle for each mode in (a) versus the angular coordinate $\phi$ (see the inset). The slopes of these curves express the density $\left(l_{\mathrm{TPC}}\right)$ of the topological Pancharatnam charge $\mathcal{C}_{\mathrm{TP}}$.

waveguide. In fact, for vector vortices, the discrete charge can reappear upon the introduction of a topological Pancharatnam charge (TPC).

\section{Topological Pancharatnam charge}

Here, using the Pancharatnam connection, the phase for any field $|a(x, y)\rangle=\left[E_{x}(x, y), E_{y}(x, y)\right]^{\mathrm{T}}$ can be written as

$$
\psi_{\mathrm{P}+}=\arg \left(\left\langle e_{+} \mid a\right\rangle\right),
$$

where the left circularly polarized field $\left|e_{+}\right\rangle=(1 / \sqrt{2})[1, i]^{\mathrm{T}}$ is used as the reference field. Similarly to scalar vortex beams, we can define the TPC for vector vortices as [46-48]

$$
\mathcal{C}_{\mathrm{TP}}=\frac{1}{2 \pi} \oint_{C} l_{\mathrm{TPC}} \mathrm{d} \phi=\frac{1}{2 \pi} \oint_{C} \frac{\partial \psi_{\mathrm{P}+}}{\partial \phi} \mathrm{d} \phi,
$$

where $\phi$ is the angular coordinate in the transverse plane, $l_{\mathrm{TPC}}=\partial \psi_{\mathrm{P}+} / \partial \phi$ is the density of $\mathcal{C}_{\mathrm{TP}}$, and $C$ is a circular path around the beam center.

With the phase definition in Eq. (4), the phase fronts for the fields in Fig. 1(a) are calculated and displayed in Fig. 2(a). To demonstrate more clearly, Fig. 2(b) shows the $\phi$ dependence of $\psi_{\mathrm{P} \pm}$ along the path of the cyan circle shown in Figs. 1(a) and 2 (a). It is easy to read that $\mathcal{C}_{\mathrm{TP}}$ equals 1 and -1 for modes $\mathrm{TE}_{01}$ and $\mathrm{TE}_{10}$, respectively. The magnitude of $\mathcal{C}_{\mathrm{TP}}$ is determined by the mode azimuthal order (node number) stemming from topological protection [46]. Then the $\mathcal{C}_{\mathrm{TP}}$ of their superposition field $\mathbf{E}^{\text {sp }}$ also can take a value of 1 or -1 depending on $\{\alpha, \delta \varphi\}$. In Fig. $2, \mathcal{C}_{\mathrm{TP}}=1$ for $\mathbf{E}^{\mathrm{sp}}$ under the condition of $\{\alpha, \delta \varphi\}=\{0.5, \pi / 2\}$. Note that all superposed fields have the same TPC magnitude (i.e., $\left|\mathcal{C}_{\mathrm{TP}}\right|=1$ ), but the TPC densities $\left(l_{\mathrm{TPC}}\right)$ are different, which can be evaluated by the slope $\left(\partial \psi_{\mathrm{P}+} / \partial \phi\right)$ of the curves in Fig. 2(b). For $\mathrm{TE}_{01}$ and $\mathrm{TE}_{10}, l_{\mathrm{TPC}}$ is varied along $\phi$ and the maximal magnitudes of 
$l_{\mathrm{TPC}}$ are reached at the nodes of the fields. While for $\mathbf{E}^{\mathrm{SP}}$ at $\{\alpha, \delta \varphi\}=\{0.5, \pi / 2\}, l_{\mathrm{TPC}}$ is smoother and undulates slightly around 1 versus $\phi$.

Figure 1(b) shows that the average canonical OAM charge can be tuned from -0.87 to 0.87 , which means that the maximal magnitude is 0.87 . It clearly shows that the maximum achievable OAM charge (equal to 0.87 ) is not equal to the TPC (equal to 1 ). This indicates that the topological Pancharatnam charge is incomplete to characterize the OAM charge for such a vector vortex. So one question to ask is how transverse confinement determines the maximal magnitude of the average canonical OAM charge in a silicon channel waveguide. Moreover, is it possible to reach 1 , which exactly equals the mode azimuthal order (i.e., $\mathcal{C}_{\mathrm{TP}}$ ) of $\mathrm{TE}_{01} / \mathrm{TE}_{10}$ ? To better appreciate these relations, we make a systematic study of the OAM and SAM in a silicon channel waveguide via sweeping structure parameters and reveal their dependencies in the following section.

\section{THE MAXIMUM ACHIEVABLE OAM CHARGE}

\section{A. AM map in the structure-parameter space}

For such a simple structure as the $\mathrm{Si} / \mathrm{SiO}_{2}$ channel waveguide, there are two parameters, silicon-core width $w_{\mathrm{Si}}$ and height $h_{\mathrm{Si}}$ [see Fig. 1(a)], for tailoring eigenmodes. The results for $\left\{w_{\mathrm{Si}}, h_{\mathrm{Si}}\right\}$ dependence of the maximum of the average canonical OAM and SAM charges are demonstrated straightforwardly in Fig. 3. To obtain the results shown in Fig. 3, we first sweep $\left\{w_{\mathrm{Si}}, h_{\mathrm{Si}}\right\}$ and get the corresponding modes of $\mathrm{TE}_{01}$ and $\mathrm{TE}_{10}$. Owing to the equivalent roles of $w_{\mathrm{Si}}$ and $h_{\mathrm{Si}}$ and consideration of TE modes, we just sweep the condition $h_{\mathrm{Si}} \leqslant w_{\mathrm{Si}}$. Then $\mathbf{E}^{\mathrm{sp}}$ can be superposed for different $\{\alpha, \delta \varphi\}$ by Eq. (3) and used for calculating the average canonical OAM and SAM charges with Eqs. (1) and (2). For each set $\left\{w_{\mathrm{Si}}, h_{\mathrm{Si}}\right\}$, the maximum charges can be filtered and are plotted in Figs. 3(a) and 3(b) for the OAM and SAM parts, respectively. It is noteworthy that, generally, the superposed vortex beams are not stable due to the different propagation constants of $\mathrm{TE}_{01}$ and $\mathrm{TE}_{10}$, however, in this work we would like to explore the maximum achievable OAM charge for superposition of $\mathrm{TE}_{01}$ and $\mathrm{TE}_{10}$ in the channel waveguide. Of course, a special case is that a stable vortex beam can be realized with a degenerate $\mathrm{TE}_{01}$ and $\mathrm{TE}_{10}$, which is shown by the dashed red curve in Fig. 3. For brevity in the following text, the maximum achievable charges of canonical OAM and SAM are referred to as the OAM and SAM charges, respectively, unless otherwise noted. From Fig. 3(a), on the whole, it can be found that the OAM charge increases as the width $\left(w_{\mathrm{Si}}\right)$ and aspect ratio $\left(w_{\mathrm{Si}} / h_{\mathrm{Si}}=\left[h_{\mathrm{Si}} / w_{\mathrm{Si}}\right]^{-1}\right)$ of the silicon core increase. In contrast, the SAM charge is inversely proportional to the two factors as shown in Fig. 3(b). The larger $w_{\mathrm{Si}}$ means weaker confinement of modes, which trends to the paraxial limit. The confinement leads to hybrid eigenmodes, which possess a space-variant state of polarization (SOP). When $w_{\mathrm{Si}} / h_{\mathrm{Si}}$ approaches 1 , the overlap between the $\mathrm{TE}_{01}$ and $\mathrm{TE}_{10}$ fields becomes large and causes strong mixing of the SOP in the superposition. In general, an intense space-variant SOP promotes the SAM charge and degrades the OAM charge, which can be supported phenomenologically by comparing
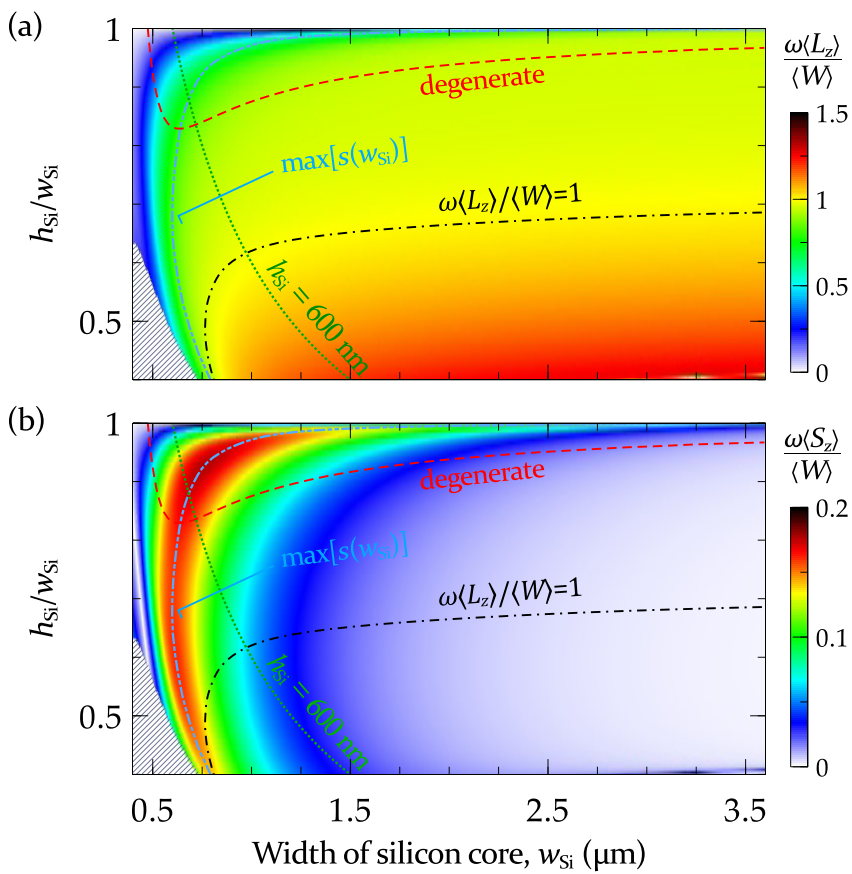

FIG. 3. Dependence of the maximum achievable charges of the (a) OAM and (b) SAM on the waveguide structure parameters $w_{\mathrm{Si}}$ and $h_{\mathrm{Si}}$. In the bottom-left corner of the graphs, the data cannot be accessed due to the cutoff of $\mathrm{TE}_{01}$. We set $s\left(w_{\mathrm{Si}}\right)=\omega\left\langle S_{z}\right\rangle /\langle W\rangle$ as a function of $w_{\mathrm{Si}}$ and plot $\max \left[s\left(w_{\mathrm{Si}}\right)\right]$ versus $h_{\mathrm{Si}}$ with the light-blue dash-dot-dotted line. The dashed red curve expresses the degenerate condition of $\mathrm{TE}_{01}$ and $\mathrm{TE}_{10}$, whose propagation constants are the same for structure parameters picked from this curve. The dotted green curve denotes a line of structure parameters with a fixed height $h_{\mathrm{Si}}=600 \mathrm{~nm}$. The dash-dotted black curve is a contour line for which the OAM charge equals 1 .

Figs. 3(a) and 3(b). In the following subsections, we further reveal the specific relation between OAM and confinement by investigating three special cases - the degenerate condition, a varying aspect ratio with a fixed height, $h_{\mathrm{Si}}=600 \mathrm{~nm}$, and an OAM charge equal to 1 -which are shown by three different curves in Fig. 3.

\section{B. Average transverse wave vectors}

To characterize the degree of mode confinement, a transverse wave vector is introduced in the spatial frequency domain. In order to obtain the transverse wave vector, we first perform Fourier transforms on $\mathcal{E}_{x}=\operatorname{Re}\left[E_{x}(x, y)\right]$ and $\mathcal{E}_{y}=\operatorname{Re}\left[E_{y}(x, y)\right]$ and generate two-dimensional maps $\mathcal{F}\left(\mathcal{E}_{x}\right)$ and $\mathcal{F}\left(\mathcal{E}_{y}\right)$ as a function of the transverse wave vector $\mathbf{k}_{\perp}=$ $\left(k_{x}, k_{y}\right)$, respectively. Then the wave vectors corresponding to the maximal magnitudes of $\mathcal{F}\left(\mathcal{E}_{x}\right)$ and $\mathcal{F}\left(\mathcal{E}_{y}\right)$ read as

$$
\mathbf{k}_{\perp}^{x F}=\left.\mathbf{k}_{\perp}\right|_{\left.\max \left[\mid \mathcal{F}\left(\mathcal{E}_{x}\right)\right]\right]}, \quad \mathbf{k}_{\perp}^{y F}=\left.\mathbf{k}_{\perp}\right|_{\max \left[\left|\mathcal{F}\left(\mathcal{E}_{y}\right)\right|\right]} ;
$$

furthermore, the two wave vectors are weighted by their electric components and the average transverse wave vector is expressed as

$$
\overline{\mathbf{k}}_{\perp}=\left(\bar{k}_{x}, \bar{k}_{y}\right)=\left\langle\left|E_{x}\right|\right\rangle \mathbf{k}_{\perp}^{x F}+\left\langle\left|E_{y}\right|\right\rangle \mathbf{k}_{\perp}^{y F} ;
$$




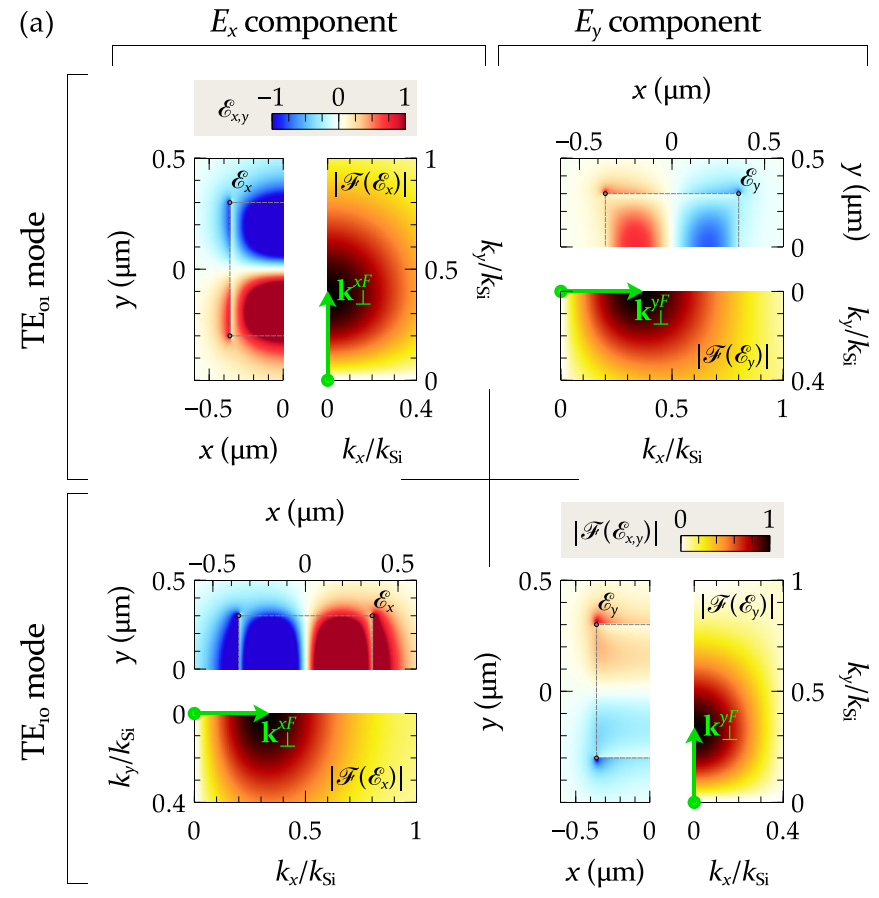

(b)

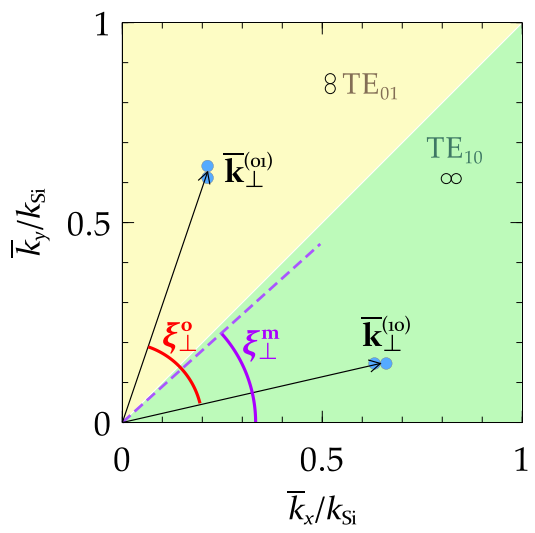

FIG. 4. Average transverse wave vectors of $\mathrm{TE}_{01}$ and $\mathrm{TE}_{10}$. (a) Fourier transform is performed on each component of the transverse electric fields of $\mathrm{TE}_{01}$ and $\mathrm{TE}_{10}$. Using the calculated $2 \mathrm{D}$ maps in the spatial frequency domain, the transverse wave vectors $\left(\mathbf{k}_{\perp}^{x F}\right.$ and $\mathbf{k}_{\perp}^{y F}$ ) corresponding to the maximum can be extracted. (b) The average transverse wave vectors $\left(\overline{\mathbf{k}}_{\perp}^{(01)} / \overline{\mathbf{k}}_{\perp}^{(10)}\right)$ of $\mathrm{TE}_{01} / \mathrm{TE}_{10}$ are calculated and depicted in $\overline{\mathbf{k}}_{\perp}$ space, where the related opening angle $\left(\xi_{\perp}^{\circ}\right)$ and angle of the median $\left(\xi_{\perp}^{\mathrm{m}}\right)$ are also shown.

finally, to eliminate confusion of the arbitrary magnitude of the electric intensity, $\overline{\mathbf{k}}_{\perp}$ is constrained by $\bar{k}_{x}^{2}+\bar{k}_{y}^{2}+k_{z}^{2}=k_{\mathrm{Si}}^{2}$ with $k_{\mathrm{Si}}=2 \pi n_{\mathrm{Si}} / \lambda$. We can find that $\overline{\mathbf{k}}_{\perp}$ contains the information, given by Eqs. (6) and (7), of both the transverse confinement and proportion of $\left(E_{x}, E_{y}\right)$. Obviously, the paraxial limit corresponds to $\left|\overline{\mathbf{k}}_{\perp}\right|$ approaching 0 . For each set $\left\{w_{\mathrm{Si}}, h_{\mathrm{Si}}\right\}$, we repeat the above procedure and obtain $\overline{\mathbf{k}}_{\perp}^{(01)}$ and $\overline{\mathbf{k}}_{\perp}^{(10)}$ for $\mathrm{TE}_{01}$ and $\mathrm{TE}_{10}$, respectively, which are illustrated in Fig. 4(a) for $\left\{w_{\mathrm{Si}}, h_{\mathrm{Si}}\right\}=\{720 \mathrm{~nm}, 600 \mathrm{~nm}\}$. For $\mathrm{TE}_{01}, \mathrm{a}$ node of $E_{x}$ (the dominant electric component) appears in the $y$ direction, which shows a higher confinement along the $y$ direction and generally results in $\bar{k}_{x}<\bar{k}_{y}$ for $\overline{\mathbf{k}}_{\perp}^{(01)}$. In the limit of a perfectly pure $\mathrm{TE}_{01}, \bar{k}_{x}$ tends to 0 arising from $E_{y}=0$ and the tiny confinement along the $x$ direction. On the contrary, $\bar{k}_{x}>\bar{k}_{y}$ for $\overline{\mathbf{k}}_{\perp}^{(10)}$ and $\bar{k}_{y}$ approaches 0 in the perfectly pure limit. Thus, in the $\overline{\mathbf{k}}_{\perp}$ space plot, $\overline{\mathbf{k}}_{\perp}^{(01)}$ and $\overline{\mathbf{k}}_{\perp}^{(10)}$ are located in the top-left half and bottom-right half, respectively, which are shown in Fig. 4(b). With the two wave vectors $\overline{\mathbf{k}}_{\perp}^{(01)}$ and $\overline{\mathbf{k}}_{\perp}^{(10)}$, the opening angle between their directions and the median of their direction angles are given by

$$
\begin{gathered}
\xi_{\perp}^{\mathrm{o}}=\arccos \left(\overline{\mathbf{k}}_{\perp}^{(01)} \cdot \overline{\mathbf{k}}_{\perp}^{(10)} /\left|\overline{\mathbf{k}}_{\perp}^{(01)}\right|\left|\overline{\mathbf{k}}_{\perp}^{(10)}\right|\right), \\
\xi_{\perp}^{\mathrm{m}}=\left(\arg \left(\overline{\mathbf{k}}_{\perp}^{(01)}\right)+\arg \left(\overline{\mathbf{k}}_{\perp}^{(10)}\right)\right) / 2 .
\end{gathered}
$$

It is found that $\xi_{\perp}^{0}$ denotes the joint influence of transverse confinement on both $\mathrm{TE}_{01}$ and $\mathrm{TE}_{10}$. The magnitude of $\xi_{\perp}^{\mathrm{o}}$ is a manifestation of the joint-SOP purity of $\mathrm{TE}_{01}-\mathrm{TE}_{10}$ in superposition. The larger $\xi_{\perp}^{\circ}$ produces the better joint-SOP purity due to the large separation of $\overline{\mathbf{k}}_{\perp}^{(01)}$ and $\overline{\mathbf{k}}_{\perp}^{(10)}$ in $\overline{\mathbf{k}}_{\perp}$ space. As shown in Fig. 4(b), the median of the direction angles of $\overline{\mathbf{k}}_{\perp}^{(01)}$ and $\overline{\mathbf{k}}_{\perp}^{(10)}$ acts as a reflection (mirror-symmetry) plane for $\overline{\mathbf{k}}_{\perp}^{(01)}$ and $\overline{\mathbf{k}}_{\perp}^{(10)}$ in $\overline{\mathbf{k}}_{\perp}$ space, so $\xi_{\perp}^{\mathrm{m}}$ can provide the information on mode complementarity between $\mathrm{TE}_{01}$ and $\mathrm{TE}_{10}$. The degree of complementarity can be evaluated by comparing $\xi_{\perp}^{\mathrm{m}}$ to $\pi / 4$ (corresponding to the critical line of $\bar{k}_{x}=\bar{k}_{y}$ ). When $\xi_{\perp}^{\mathrm{m}}=\pi / 4$, there is a high complementarity in transverse the electric fields of $\mathrm{TE}_{01}$ and $\mathrm{TE}_{10}$, which means that their superposition can offer a smooth field distribution along the $\phi$ direction. The deflection of $\xi_{\perp}^{\mathrm{m}}$ to $\pi / 4$ shows the degradation of the smooth superposed field. Note that $\xi_{\perp}^{\mathrm{m}}$ is also a composite index, which manifests itself in complementarity in the distributions of both the SOP (vectorial property) and the electric intensity.

\section{Superposition with degenerate modes}

According to the structure parameters of the degenerate curve shown in Fig. 3, the degenerate propagation constant of $\mathrm{TE}_{01} / \mathrm{TE}_{10}$ and the OAM charge of their superposed fields as a function of $w_{\mathrm{Si}}$ are calculated and displayed in Fig. 5(a). The smallest cross section of the silicon core for no-cutoff degenerate $\mathrm{TE}_{01} / \mathrm{TE}_{10}$ is exactly square, where the degenerate modes have the smallest propagation constant due to the highest mode confinement. Their superposition generates a cylindrical vector beam, whose OAM and SAM charges are both 0 [see Fig. 5(a)]. Growth of $w_{\mathrm{Si}}$, which means a reduction in the mode confinement, can increase the propagation constant and the OAM charge. In the paraxial limit, the OAM charge is close to 1 and the SAM charge approaches 0 .

For the degenerate case in $\overline{\mathbf{k}}_{\perp}$ space, $\xi_{\perp}^{\text {o gradually increases }}$ from 0 to $\pi / 2$ following $w_{\mathrm{Si}}$ [see Fig. $5(\mathrm{~b})$ ], which denotes that the joint-SOP purity of $\mathrm{TE}_{01}-\mathrm{TE}_{10}$ is increased with reduced confinement. But $\xi_{\perp}^{\mathrm{m}}$ goes down from $\pi / 4$ to a certain value and increases close to $\pi / 4$ again in the paraxial limit. The maximum for the $\xi_{\perp}^{\mathrm{m}}$ deflection $\left(\left|\xi_{\perp}^{\mathrm{m}}-\pi / 4\right|\right)$ means a low complementarity between $\mathrm{TE}_{01}$ and $\mathrm{TE}_{10}$. This maximal $\xi_{\perp}^{\mathrm{m}}$ deflection, associated with a poor joint-SOP purity $\left(\xi_{\perp}^{\mathrm{o}}<\right.$ $\pi / 2$ ), induces a large mixture of SOPs in a superposed field and corresponds to the maximum of the SAM charge, which is illustrated by comparing $\omega\left\langle S_{z}\right\rangle /\langle W\rangle$ in Fig. 5(a) with $\xi_{\perp}^{\mathrm{m}}$ in Fig. 5(b). Note that, in the paraxial limit under the degenerate condition, the OAM charge tends to 1 but is always smaller 


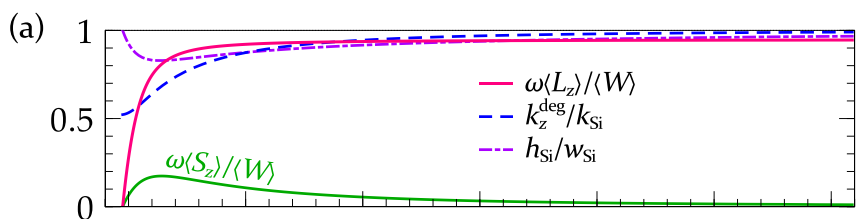

(b)

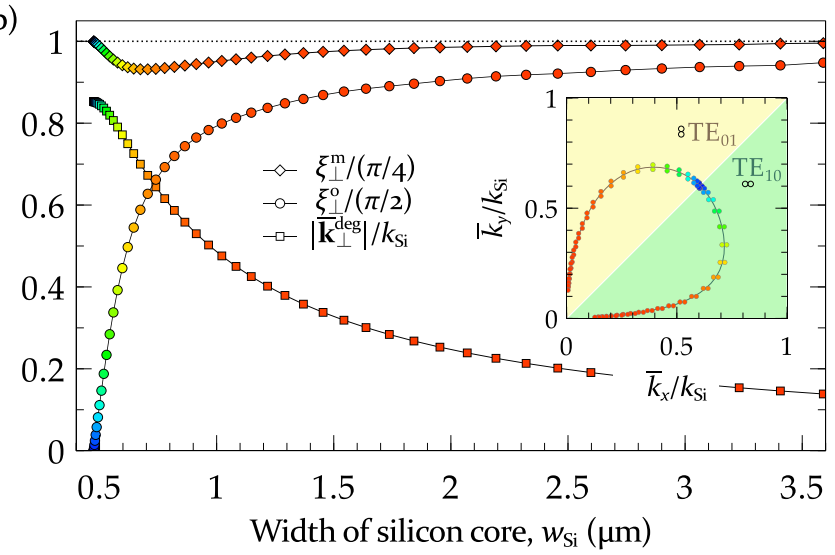

FIG. 5. AM charges and spatial frequencies of modes under the degenerate condition. (a) Silicon-core width dependence of the propagation constant and the AM charges. The guiding line of the structure parameter is redrawn as the degenerate curve shown in Fig. 3(a). (b) Silicon-core width dependence of the average transverse wave numbers, the opening angle, and the angle of the median for $\overline{\mathbf{k}}_{\perp}^{(01)}$ and $\overline{\mathbf{k}}_{\perp}^{(10)}$, whose distributions in $\overline{\mathbf{k}}_{\perp}$ space are displayed in the inset. Markers of the same color indicate calculations with identical structure parameters. For the degenerate condition, $k_{z}^{\mathrm{deg}} \equiv$ $k_{z}^{(01)}=k_{z}^{(10)}$ and $\left|\overline{\mathbf{k}}_{\perp}^{\mathrm{deg}}\right| \equiv\left|\overline{\mathbf{k}}_{\perp}^{(01)}\right|=\left|\overline{\mathbf{k}}_{\perp}^{(10)}\right|$.

than 1 , which results from the relatively high complementarity between $\mathrm{TE}_{01}$ and $\mathrm{TE}_{10}$. A further explanation is given in the following cases. The degenerate case well demonstrates that the average transverse wave vectors $\overline{\mathbf{k}}_{\perp}^{(01)}$ and $\overline{\mathbf{k}}_{\perp}^{(10)}$ can offer rich information about mode confinement, which is useful to evaluate the behaviors of the SAM and OAM of superposed fields. To further manifest this, we study a generic case of varying $w_{\mathrm{Si}}$ at $h_{\mathrm{Si}}=600 \mathrm{~nm}$.

\section{Varying $w_{\mathrm{Si}}$ at $h_{\mathrm{Si}}=600 \mathrm{~nm}$}

Figure 6(a) displays the $w_{\mathrm{Si}}$ dependence of the SAM and OAM charges in this case. Following the growth of $w_{\mathrm{Si}}$, the SAM charge increases from 0 to a certain value and decreases to 0 again, which is similar to that in the degenerate case. But the $w_{\text {Si }}$ dependence of the OAM charge is different from that in the degenerate case; the OAM charge starts at 0 , increases continually, and finally moves beyond the value of 1 . An OAM charge larger than 1 means that it can break through the mode azimuthal order of $\mathrm{TE}_{01}$ and $\mathrm{TE}_{10}$ (i.e., the OAM charge is larger than $\left|\mathcal{C}_{\mathrm{TP}}\right|$ ). In the spatial frequency domain, the related average transverse wave vectors $\overline{\mathbf{k}}_{\perp}^{(01)}$ and $\overline{\mathbf{k}}_{\perp}^{(10)}$ are displayed in Fig. 6(b). The decreasing magnitudes of $\overline{\mathbf{k}}_{\perp}^{(\overline{01})}$ and $\overline{\mathbf{k}}_{\perp}^{(10)}$ with the growth of $w_{\mathrm{Si}}$ express the mode confinements becoming weaker. Concomitantly, $\xi_{\perp}^{\circ}$ gradually grows from 0 to a constant but not $\pi / 2$, and $\xi_{\perp}^{\mathrm{m}}$ decreases from $\pi / 4$ to a certain value, goes back to nearly $\pi / 4$, then decreases again. (a)

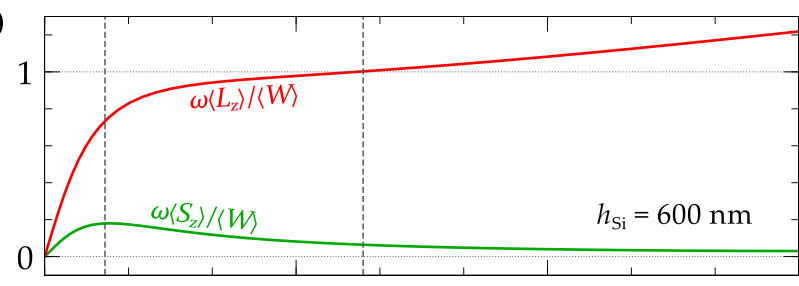

(b)

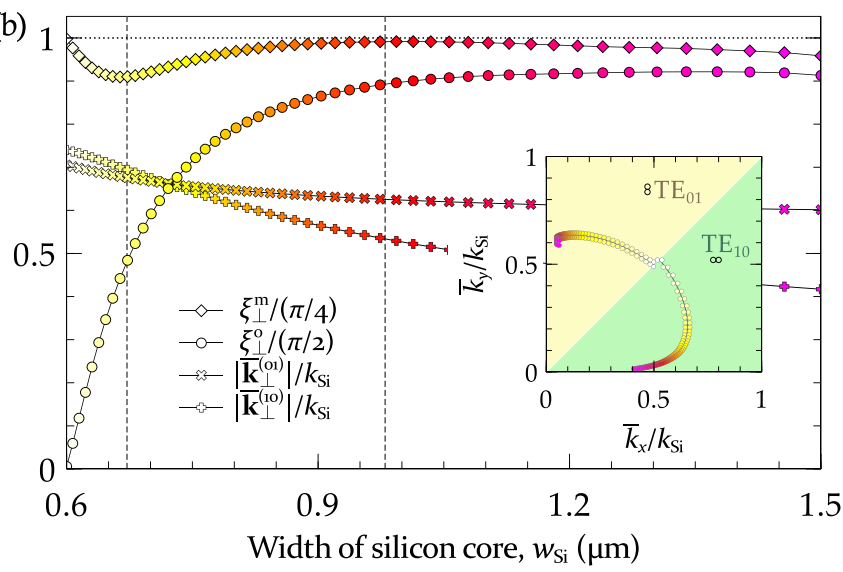

FIG. 6. AM charges and spatial frequencies of light fields at $h_{\mathrm{Si}}=600 \mathrm{~nm}$. (a) SAM and OAM charges as a function of the silicon-core width. (b) Silicon-core width dependence of the average transverse wave numbers, the opening angle, and the angle of the median for $\overline{\mathbf{k}}_{\perp}^{(01)}$ and $\overline{\mathbf{k}}_{\perp}^{(10)}$, whose distributions in $\overline{\mathbf{k}}_{\perp}$ space are displayed in the inset. Markers of the same color indicate calculations with identical structure parameters.

Comparing Figs. 6(a) and 6(b), it is found that there are two special settings of $w_{\mathrm{Si}}$, which are shown by the two vertical lines. One setting offers the maximum SAM charge in the presence of the maximal $\left|\xi_{\perp}^{\mathrm{m}}-\pi / 4\right|$, which resembles their dependence in the degenerate case. Another scenario is that the OAM charge takes a value of 1 , which corresponds to the return of $\xi_{\perp}^{\mathrm{m}} \simeq \pi / 4$. Across this setting, the OAM charge increases continually with the increment in $\left|\xi_{\perp}^{\mathrm{m}}-\pi / 4\right|$.

To provide deeper insight into the origin of the OAM, we separate the OAM density into two contributions with the approach introduced in Refs. [47,48]. For any field $|a\rangle$, the normalized Stokes vector $\mathbf{S}=\left(S_{1}, S_{2}, S_{3}\right)^{\mathrm{T}}$ can be defined by $S_{j}=\left\langle a\left|\sigma_{j}\right| a\right\rangle / I_{\mathrm{E}}(j=1,2,3)$, where $\sigma_{j}$ are the Pauli matrices and $I_{\mathrm{E}}=\langle a \mid a\rangle$ is the electric intensity. Then the Poincaré sphere can be constructed by mapping the Stokes vector $\mathbf{S}$ on Cartesian coordinates, whose azimuth $\left(\psi_{\mathrm{S}}\right)$ is calculated by

$$
\psi_{\mathrm{S}}=\arctan \left(S_{2} / S_{1}\right) / 2
$$

According to Refs. $[47,48]$, the longitudinal OAM density $j_{z}^{\mathrm{o}}$ can be expressed as

$$
\frac{j_{z}^{\mathrm{o}}}{\omega \epsilon_{0} I_{\mathrm{E}}}=\frac{\partial \psi_{\mathrm{P}+}}{\partial \phi}+\left(1-S_{3}\right) \frac{\partial \psi_{\mathrm{S}}}{\partial \phi}=l_{\mathrm{TPC}}+l_{\mathrm{SOP}} .
$$

In Eq. (11), the first term exactly corresponds to the density of the TPC given in Eq. (5) and the second term represents the contribution induced by a spatially varied SOP (termed the SOP-OAM density and denoted $l_{\mathrm{SOP}}$ ). With the ratio of angular momentum to energy that was examined by Allen [2], 
(a)

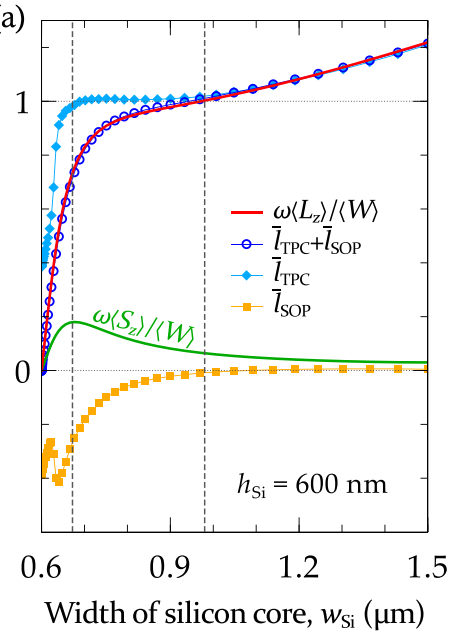

(b) $w_{\mathrm{Si}}=600 \mathrm{~nm}$
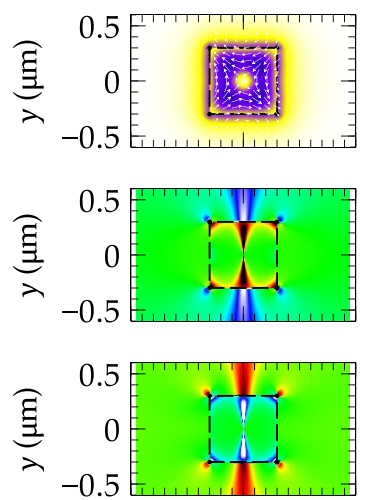

$-1$

0
$x(\mu \mathrm{m})$ (c) $w_{\mathrm{Si}}=700 \mathrm{~nm}$
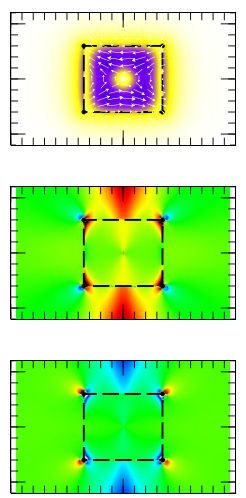

$-1$

0

$x(\mu \mathrm{m})$ (d) $w_{\mathrm{si}}=1000 \mathrm{~nm}$
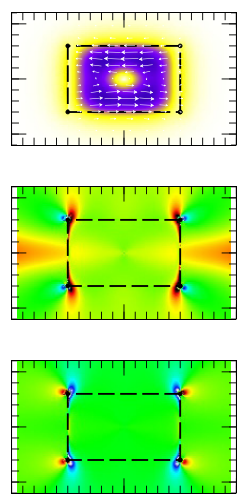

$1-1$ (e) $w_{\mathrm{Si}}=1500 \mathrm{~nm}$
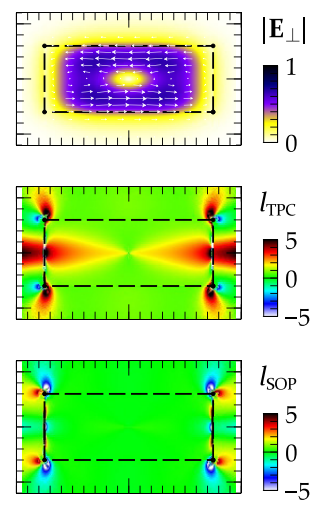

$\begin{array}{lll}0 & 1-1\end{array}$

$x(\mu \mathrm{m})$
0

$x(\mu \mathrm{m})$

FIG. 7. OAM charge contributions and densities of vortices at $h_{\mathrm{Si}}=600 \mathrm{~nm}$. (a) The average TPC-OAM charge and SOP-OAM charge and their sum as a function of the silicon-core width. Curves for the average canonical SAM and OAM charges are redrawn as those shown in Fig. 6(a). The related density calculations are demonstrated for four silicon-core widths of (b) $600 \mathrm{~nm}$, (c) $700 \mathrm{~nm}$, (d) $1000 \mathrm{~nm}$, and (e) $1500 \mathrm{~nm}$. Each of (b)-(e) has three panels: distributions of the transverse electric field (top), TPC-OAM density (middle), and SOP-OAM density (bottom). The dashed black boxes show the boundaries of the silicon-core region.

the average OAM charge can be calculated using Eq. (11) as

$$
\bar{l}=\frac{\iint j_{z}^{\mathrm{o}} r d r d \phi}{\omega \epsilon_{0} \iint I_{\mathrm{E}} r d r d \phi}=\bar{l}_{\mathrm{TPC}}+\bar{l}_{\mathrm{SOP}}
$$

where $r$ is the radial coordinate in the transverse plane, the first term is the corresponding average TPC-OAM charge, and the second term is the average SOP-OAM charge. With the use of Eq. (12), the calculated TPC- and SOP-OAM charges are plotted in Fig. 7(a) for the case of $h_{\mathrm{Si}}=600 \mathrm{~nm}$. It clearly shows that the sum of the TPC- and SOP-OAM charges matches well with the calculation for the average canonical OAM charge using Eq. (2). According to the two special settings of $w_{\mathrm{Si}}$ in Fig. 6, the charts of OAM charge contributions can be roughly divided into three stages as shown in Fig. 7(a). The first stage, at the beginning of $w_{\mathrm{Si}}$, shows that both the TPC- and the SOP-OAM charges vary strongly but offer opposite contributions. In particular, at the lowest setting, $w_{\mathrm{Si}}=600 \mathrm{~nm}$, corresponding to a square cross section, the TPC- and SOP-OAM charges are equal but opposite, which induces a total OAM charge equaling 0 . In the second stage, the variation of the TPC-OAM charge is moderated and the SOP-OAM charge approaches 0 . In the third stage, the SOP-OAM charge remains 0 , while the TPC-OAM charge increases constantly from 1. Comparing with Fig. 6, we find that the SOP-OAM evolves strongly in the first and second stages, where both the poor joint-SOP purity $\left(\xi_{\perp}^{\mathrm{o}}<\pi / 2\right)$ and the degradation of complementarity (mainly manifested in the SOP distributions) occur in tightly confined fields. In the third stage, the degradation of complementarity (mainly manifested in the distributions of the transverse electric intensity) appears again due to the high aspect ratio of the waveguide and leads to an increment in the TPC-OAM charge.

To further demonstrate their dependencies, the transverse electric intensity, TPC-OAM density, and SOP-OAM density are calculated by Eq. (11) and displayed in Figs. 7(b)-7(e) for four settings of $w_{\mathrm{Si}}$. As shown in the top panels in
Figs. 7(b)-7(e), the SOP purities are enhanced and the aspect ratio of the intensity distribution increases following an increment in $w_{\mathrm{Si}}$. These indicate that, with regard to complementary symmetry about the $x=y$ (i.e., $45^{\circ}$ ) line, the reflection on the intensity distribution is reduced while the similarity of the SOP distribution is roughly promoted. In the middle and bottom panels in Figs. 7(b)-7(e), we focus on the distributions within the dashed black boxes since they provide the major contributions to the weighted average OAM charges. The SOP-OAM density decreases and becomes 0 with the growth of $w_{\mathrm{Si}}$, which is shown in the bottom panels in Figs. 7(b)-7(e). But for the TPC-OAM density, some local densities are very high at small $w_{\mathrm{Si}}$ [see middle panel in Fig. 7(b)], and the density distribution becomes smooth at large $w_{\mathrm{Si}}$ [Figs. 7(c) and 7(d)], then some local densities increase to very high values again at a larger $w_{\mathrm{Si}}[\mathrm{Fig}$. 7(e)]. In high confinement (i.e., with a small $w_{\mathrm{Si}}$ ), the SOP-OAM contribution counteracting the TPC-OAM contribution makes the total OAM charge smaller than 1. In weak confinement, the total OAM charge can achieve a value larger than 1 since the SOP-OAM density becomes almost 0 . This result shows that the OAM charge can reach beyond the mode azimuthal order of $\mathrm{TE}_{01}$ and $\mathrm{TE}_{10}$. In the case of $h_{\mathrm{Si}}=600 \mathrm{~nm}$, we can find that the $\xi_{\perp}^{\mathrm{m}}$ deflection (i.e., degradation of complementarity) induces a large SOP-OAM contribution in high confinement, while it almost wholly converts to the TPCOAM contribution in weak confinement. In particular, the TPC-OAM charge arising from the degradation of complementarity can promote the total OAM charge beyond the mode azimuthal order. Due to the particularity and typicality of an OAM charge equal to 1 , we show the behaviors under this condition in the next subsection.

\section{E. Realizing $\omega\left\langle L_{z}\right\rangle /\langle W\rangle=1$}

With the help of the contour map of OAM charge shown in Fig. 3(a), it is easy to extract the isoline of $\omega\left\langle L_{z}\right\rangle /\langle W\rangle=1$ 


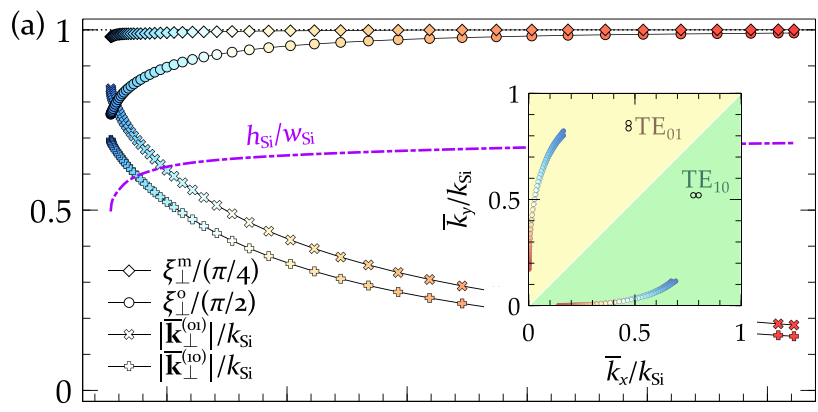

(b)

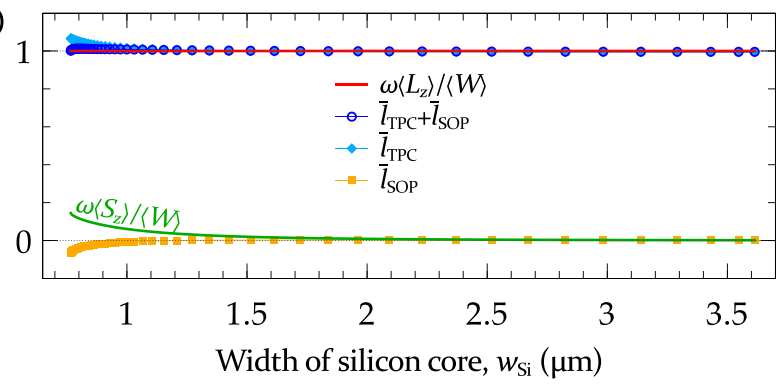

(c) $w_{\mathrm{Si}}=800 \mathrm{~nm}$

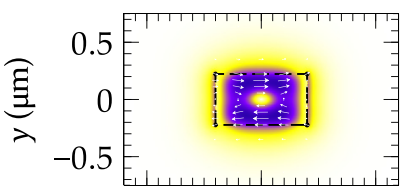

(d) $w_{\mathrm{Si}}=3000 \mathrm{~nm}$
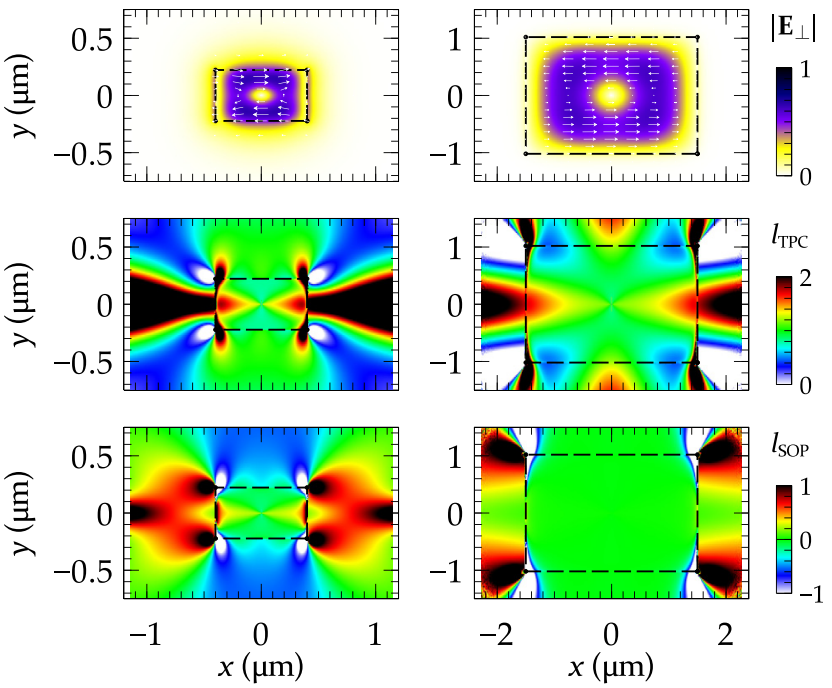

FIG. 8. AM charges, spatial frequencies, and charge densities of beams for the scenario of the OAM charge equaling 1. (a) Siliconcore width dependence of the average transverse wave numbers, the opening angle, and the angle of the median for $\overline{\mathbf{k}}_{\perp}^{(01)}$ and $\overline{\mathbf{k}}_{\perp}^{(10)}$, whose distributions in $\overline{\mathbf{k}}_{\perp}$ space are displayed in the inset. Markers of the same color indicate calculations with identical structure parameters. The guiding line of the structure parameter is redrawn as that shown in Fig. 3(a). (b) The average canonical SAM and OAM charges and the average TPC-OAM and SOP-OAM charges (and the sum) as a function of the silicon-core width. The related density calculations are demonstrated for two silicon-core widths: (c) $800 \mathrm{~nm}$ and (d) $3000 \mathrm{~nm}$. Each of (c) and (d) has three panels: distributions of the transverse electric field (top), TPC-OAM density (middle), and SOP-OAM density (bottom). The dashed black boxes show the boundaries of the silicon-core region.

and derive the structure parameters. With the derived structure relation and associated eigenmodes, we calculate the related parameters in the spatial frequency domain, which are shown in Fig. 8(a). It is found that $\xi_{\perp}^{\circ}$ starting from a certain value finally approaches $\pi / 2$. And $\xi_{\perp}^{\mathrm{m}}$ remains $\pi / 4$ well for almost all the parameters except for the deeply confined fields at an initially small $w_{\mathrm{Si}}$. This means that a small $\xi_{\perp}^{\mathrm{m}}$ deflection with a not-so-good joint-SOP purity occurs at very high mode confinement, where the SOP is mixed slightly and the SOPOAM contribution is involved in the total OAM charge. The corresponding separated OAM contributions are calculated and displayed in Fig. 8(b). As expected, apart from the beginning of $w_{\mathrm{Si}}$, the TPC-OAM and SOP-OAM charges remain almost-constant. At the beginning of $w_{\mathrm{Si}}$, the TPC-OAM and SOP-OAM charges show some small shifting but they can compensate each other to keep the total OAM charge value 1. And the expected small rise in the SAM charge also can be found at the beginning. To further illustrate, the related density distributions are demonstrated for two settings of $w_{\mathrm{Si}}$ in Figs. 8(c) and 8(d). For a low setting, $w_{\mathrm{Si}}=800 \mathrm{~nm}$, some local SOP-OAM densities are not equal to 0 [see bottom panel in Fig. 8(c)]. But this will disappear in the larger setting of $w_{\mathrm{Si}}=3000 \mathrm{~nm}$ [Fig. 8(d)]. However, the TPC-OAM density is not smooth in distributions and can reach values larger than 1 for both settings [see middle panel in Figs. 8(c) and 8(d)]. But the local TPC-OAM density with a large amount appears farther away from the region of high electric intensity at larger $w_{\mathrm{Si}}$ than at small $w_{\mathrm{Si}}$. In general, there is a series of options of structure parameters to generate an OAM charge equaling 1 and these options almost represent the highest complementarity between $\mathrm{TE}_{01}$ and $\mathrm{TE}_{10}$ in the whole parameter space.

\section{F. The structure-parameter space is divided into three regimes}

Based on the preceding demonstrations, we can divide the full parameter space into three regimes with two curves$\omega\left\langle L_{z}\right\rangle /\langle W\rangle=1$ and $\max \left[s\left(w_{\mathrm{Si}}\right)\right]$ with $s\left(w_{\mathrm{Si}}\right)=\omega\left\langle S_{z}\right\rangle /\langle W\rangle-$ as a function of $w_{\mathrm{Si}}$, which are plotted in Fig. 3. The curve of $\max \left[s\left(w_{\mathrm{Si}}\right)\right]$ means that the degree of SOP mixing is maximal in superposed fields, which also implies that the $\xi_{\perp}^{\mathrm{m}}$ deflection reaches the extremum. Before this extremum (lower side of $w_{\mathrm{Si}}$ ), both the TPC- and the SOP-OAM charges vary strongly and involve deeply in the contribution to OAM, but their sum (i.e., the total OAM charge) stays at a low value $(<0.8)$. In the upper end of this extremum, the variations of the TPCand SOP-OAM charges become moderate and the SOP-OAM charge approaches 0 following the growth of $w_{\mathrm{Si}}$. Then reaching the curve of $\omega\left\langle L_{z}\right\rangle /\langle W\rangle=1, \xi_{\perp}^{\mathrm{m}}$ returns to almost $\pi / 4$, which denotes a high complementarity between $\mathrm{TE}_{01}$ and $\mathrm{TE}_{10}$. Further beyond $\omega\left\langle L_{z}\right\rangle /\langle W\rangle=1, \xi_{\perp}^{\mathrm{m}}$ moves away from $\pi / 4$ again due to the degradation of complementarity in the electric intensity distributions. In this regime, the total OAM charge $(>1)$ grows constantly, resulting from the increment in the TPC-OAM charge, whereas the SOP-OAM charge remains almost 0 . In general, the paraxial limit can be achieved by increasing $w_{\mathrm{Si}}$, where the three curves of $\max \left[s\left(w_{\mathrm{Si}}\right)\right]$, degenerate condition, and $\omega\left\langle L_{z}\right\rangle /\langle W\rangle=1$ will merge in this limit.

\section{CONCLUSION}

This work has studied the SAM and OAM of highly confined vertex fields in silicon channel waveguides, which are generated by the superposition of $\mathrm{TE}_{01}$ and $\mathrm{TE}_{10}$ modes. Compared with cylindrical fibers, there are similar features for the SAM and the intrinsic longitudinal OAM: In high 
confinement, the space-variant SOP modes induce a nonzero SAM charge and degrade the TPC-OAM charge; in the paraxial limit, the pure SOP modes give a zero SAM charge and promise that the total OAM charge is only contributed by the TPC-OAM. However, there are also several significant differences:

(i) In cylindrical fibers, there are eigenmodes that carry OAM, even though they are not easily excited by general optical sources. The reason is that the rotation invariant guarantees the support of spatially twisted light. In channel waveguides, there is no rotation invariant, so a stable twisted light cannot be supported in principle. But the $x$ - and $y$-mirror symmetries allow the channel waveguide to support the vertex field upon the occurrence of accidental degeneracy (e.g., degeneracy between $\mathrm{TE}_{01}$ and $\mathrm{TE}_{10}$ ). This requirement is critical and ideal since the stability of the vortex greatly depends on the wavelength of light and structure parameters. Under the degenerate condition, the relation $\left\langle P_{z}\right\rangle /\langle W\rangle=\beta / \omega$ also holds for superposed modes in channel waveguides. In general conditions, the OAM charge of vortex fields cannot be preserved during propagation owing to the different propagation constants of $\mathrm{TE}_{01}$ and $\mathrm{TE}_{10}$. The spatial beating generates a periodically varied OAM charge, whose "amplitude" is the maximum achievable OAM charge investigated in this work.

(ii) In cylindrical fibers, the AM densities of twisted eigenmodes are uniform along the $\phi$ direction due to the rotation invariant, which makes the total AM charge exactly equal to the mode azimuthal order (equivalent to $\mathcal{C}_{\mathrm{TP}}$ ). The difference between high and low confinements is just the different proportions between SAM and OAM. In channel waveguides, the distributions of AM densities just satisfy the reflection symmetry, which implies that the uniformity of density along the $\phi$ direction is not necessary. This means that the total AM charge can break through the restriction of $\mathcal{C}_{\mathrm{TP}}$. The total AM charge can be smaller and even larger than $\mathcal{C}_{\mathrm{TP}}$.

(iii) For the simplest step-index fibers, there is only one structure parameter, the radius of the core. The radius solely determines the mode confinement, which also means the proportion between SAM and OAM is locked by this radius. For channel waveguides, there are two independent parameters, $w_{\mathrm{Si}}$ and $h_{\mathrm{Si}}$, which allow adjustment of the proportion between $\mathrm{SAM}$ and OAM by changing the aspect ratio $w_{\mathrm{Si}} / h_{\mathrm{Si}}$ with a comparable confinement.

In the separation of the OAM charge, we used the approach proposed in Refs. $[47,48]$. It should be noted that this is just an "empirical" application because the approximations of the paraxial limit are used in Eq. (11). But the calculation results matched well with the results calculated directly by fields using Eq. (2). The remarkable application of this approach is that the accurate OAM charge can be measured with two detectable parameters $[47,49]$. Here, we find that it is also helpful to analyze the contributions of OAM and manipulate AM densities in design.

In summary, we have studied the SAM and OAM of light in silicon channel waveguides. A vortex beam carrying OAM can be generated by a superposition of the $\mathrm{TE}_{01}$ and $\mathrm{TE}_{10}$ modes. The longitudinal OAM of the superposed vortex beam is intrinsic and mediated by the superposition coefficient. The SAM and OAM of the generated vortex fields are strongly coupled in silicon waveguides due to the transverse confinement. To characterize the confinement, we proposed the average transverse wave vector and saw a good correspondence with the hybridization of SAM and OAM. Combining the SAM and OAM charges, the whole structure parameter space can be divided into three regimes. Meanwhile, separation of the OAM was achieved with the use of the topological Pancharatnam charge and Stokes parameters. The investigation permits increased functionality, applicability, and design freedom of the angular momentum of optical fields in silicon channel waveguides.

\section{ACKNOWLEDGMENT}

The author thanks Dr. J. S. Tsai for his encouragement and support.

\section{APPENDIX: DISPERSION CURVE OF LIGHT IN A SILICON CHANNEL WAVEGUIDE}

In a dielectric slab waveguide, perfect transverse electric (TE) and transverse magnetic (TM) modes are supported, since there is only one direction confinement [50]. Typically, assuming that the modes are confined in the $y$ direction and propagate along the $z$ direction, the TE modes have the components $\left\{E_{x}, H_{y}, H_{z}\right\}$, while the TM modes possess the components $\left\{H_{x}, E_{y}, E_{z}\right\}$. However, for a channel waveguide, there are only hybrid eigenmodes due to confinements from both the $x$ and the $y$ directions. This means that all six components are possessed by all eigenmodes. To classify these modes, we assume that the quasi-TE modes are the mode fields that have the major components $\left\{E_{x}, H_{y}, H_{z}\right\}$, and the quasi-TM modes are the mode fields that have the major components $\left\{H_{x}, E_{y}, E_{z}\right\}$. That is, viewed from the transverse electric components (i.e., $E_{x}$ and $E_{y}$ ), the eigenmodes with major $E_{x}$ are denoted quasi-TE modes and the eigenmodes with major $E_{y}$ are denoted quasi-TM modes, which are usually called TE and TM modes, respectively, for short. Further classification is performed by numbering the
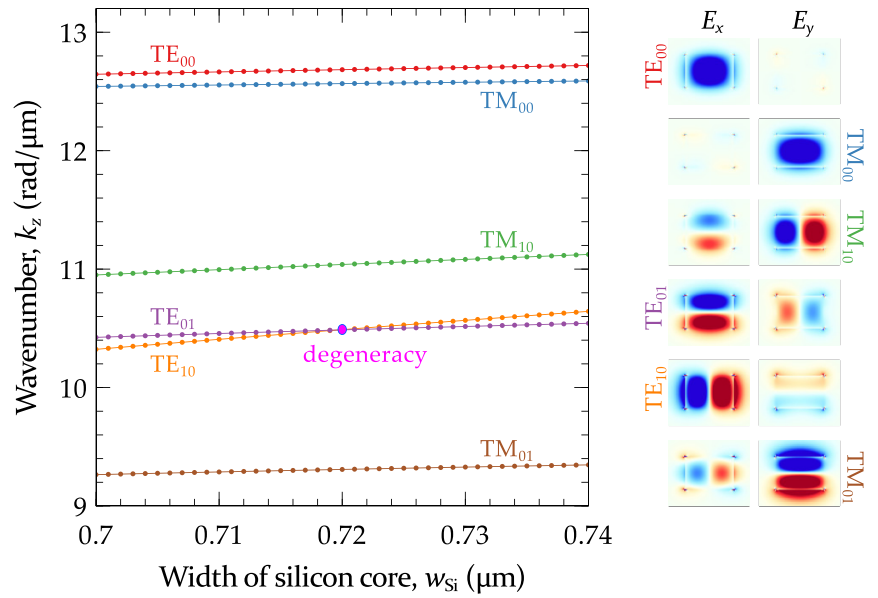

FIG. 9. Structural dispersion curve for a silicon channel waveguide for a fixed height of $600 \mathrm{~nm}$ at a wavelength of $1500 \mathrm{~nm}$. The insets at the right schematically show the amplitude distributions of $E_{x}$ and $E_{y}$ for the lowest six modes. 
nodes along the $x$ and $y$ directions, which is on $E_{x}$ for TE modes and $E_{y}$ for TM modes. Figure 9 displays the lowest six eigenmodes for a silicon channel waveguide via numerical calculations [51]. Meanwhile, each mode is labeled with the described classification approach. In the calculations, we sweep $w_{\mathrm{Si}}$ around $720 \mathrm{~nm}$ with a fixed $h_{\mathrm{Si}}=600 \mathrm{~nm}$ at a wavelength of $1550 \mathrm{~nm}$. It is found that the $\mathrm{TE}_{01}$ and $\mathrm{TE}_{10}$ modes are degenerate at $w_{\mathrm{Si}}=720 \mathrm{~nm}$.
[1] M. Padgett, J. Courtial, and L. Allen, Light's orbital angular momentum, Phys. Today 57(5), 35 (2004).

[2] L. Allen, M. W. Beijersbergen, R. J. C. Spreeuw, and J. P. Woerdman, Orbital angular momentum of light and the transformation of Laguerre-Gaussian laser modes, Phys. Rev. A 45, 8185 (1992).

[3] S. J. Van Enk and G. Nienhuis, Spin and orbital angular momentum of photons, Europhys. Lett. 25, 497 (1994).

[4] K. Y. Bliokh, F. J. Rodríguez-Fortuño, F. Nori, and A. V. Zayats, Spin-orbit interactions of light, Nat. Photon. 9, 796 (2015).

[5] N. Yu, P. Genevet, M. A. Kats, F. Aieta, J.-P. Tetienne, F. Capasso, and Z. Gaburro, Light propagation with phase discontinuities: Generalized laws of reflection and refraction, Science 334, 333 (2011)

[6] A. Arbabi, Y. Horie, M. Bagheri, and A. Faraon, Dielectric metasurfaces for complete control of phase and polarization with subwavelength spatial resolution and high transmission, Nat. Nanotechnol. 10, 937 (2015).

[7] R. C. Devlin, A. Ambrosio, N. A. Rubin, J. P. B. Mueller, and F. Capasso, Arbitrary spin-to-orbital angular momentum conversion of light, Science 358, 896 (2017).

[8] L. Marrucci, C. Manzo, and D. Paparo, Optical Spin-ToOrbital Angular Momentum Conversion in Inhomogeneous Anisotropic Media, Phys. Rev. Lett. 96, 163905 (2006).

[9] X.-L. Wang, X.-D. Cai, Z.-E. Su, M.-C. Chen, D. Wu, L. Li, N.-L. Liu, C.-Y. Lu, and J.-W. Pan, Quantum teleportation of multiple degrees of freedom of a single photon, Nature 518, 516 (2015).

[10] Z.-Y. Zhou, S.-L. Liu, Y. Li, D.-S. Ding, W. Zhang, S. Shi, M.-X. Dong, B.-S. Shi, and G.-C. Guo, Orbital Angular Momentum-Entanglement Frequency Transducer, Phys. Rev. Lett. 117, 103601 (2016).

[11] T. Stav, A. Faerman, E. Maguid, D. Oren, V. Kleiner, E. Hasman, and M. Segev, Quantum entanglement of the spin and orbital angular momentum of photons using metamaterials, Science 361, 1101 (2018).

[12] S. M. Barnett, Optical angular-momentum flux, J. Opt. B 4, S7 (2002).

[13] Y. Zhao, J. S. Edgar, G. D. M. Jeffries, D. McGloin, and D. T. Chiu, Spin-To-Orbital Angular Momentum Conversion in a Strongly Focused Optical Beam, Phys. Rev. Lett. 99, 073901 (2007).

[14] Z. Bomzon and M. Gu, Space-variant geometrical phases in focused cylindrical light beams, Opt. Lett. 32, 3017 (2007).

[15] C. N. Alexeyev, A. N. Alexeyev, B. P. Lapin, G. Milione, and M. A. Yavorsky, Spin-orbit-interaction-induced generation of optical vortices in multihelicoidal fibers, Phys. Rev. A 88, 063814 (2013).

[16] P. Gregg, P. Kristensen, and S. Ramachandran, Conservation of orbital angular momentum in air-core optical fibers, Optica 2 , 267 (2015)
[17] S. Ramachandran and P. Kristensen, Optical vortices in fiber, Nanophotonics 2, 455 (2013).

[18] F. Le Kien, T. Busch, V. G. Truong, and S. Nic Chormaic, Higher-order modes of vacuum-clad ultrathin optical fibers, Phys. Rev. A 96, 023835 (2017).

[19] M. F. Picardi, K. Y. Bliokh, F. J. Rodríguez-Fortuño, F. Alpeggiani, and F. Nori, Angular momenta, helicity, and other properties of dielectric-fiber and metallic-wire modes, Optica $\mathbf{5}$, 1016 (2018).

[20] K. Y. Bliokh, A. Niv, V. Kleiner, and E. Hasman, Geometrodynamics of spinning light, Nat. Photon. 2, 748 (2008).

[21] C. C. Leary, M. G. Raymer, and S. J. van Enk, Spin and orbital rotation of electrons and photons via spin-orbit interaction, Phys. Rev. A 80, 061804(R) (2009).

[22] D. L. P. Vitullo, C. C. Leary, P. Gregg, R. A. Smith, D. V. Reddy, S. Ramachandran, and M. G. Raymer, Observation of Interaction of Spin and Intrinsic Orbital Angular Momentum of Light, Phys. Rev. Lett. 118, 083601 (2017).

[23] D. R. Abujetas and J. A. Sánchez-Gil, Spin angular momentum of guided light induced by transverse confinement and intrinsic helicity, ACS Photon. 7, 534 (2020).

[24] J. Petersen, J. Volz, and A. Rauschenbeutel, Chiral nanophotonic waveguide interface based on spin-orbit interaction of light, Science 346, 67 (2014).

[25] K. Y. Bliokh and F. Nori, Transverse and longitudinal angular momenta of light, Phys. Rep. 592, 1 (2015).

[26] R. J. Coles, D. M. Price, J. E. Dixon, B. Royall, E. Clarke, P. Kok, M. S. Skolnick, A. M. Fox, and M. N. Makhonin, Chirality of nanophotonic waveguide with embedded quantum emitter for unidirectional spin transfer, Nat. Commun. 7, 11183 (2016).

[27] R. Soref, The past, present, and future of silicon photonics, IEEE J. Sel. Top. Quant. Electron. 12, 1678 (2006).

[28] D. Thomson, A. Zilkie, J. E. Bowers, T. Komljenovic, G. T. Reed, L. Vivien, D. Marris-Morini, E. Cassan, L. Virot, J.-M. Fédéli, J.-M. Hartmann, J. H. Schmid, D.-X. Xu, F. Boeuf, P. O’Brien, G. Z. Mashanovich, and M. Nedeljkovic, Roadmap on silicon photonics, J. Opt. 18, 073003 (2016).

[29] X. Xiong, C.-L. Zou, X.-F. Ren, and G.-C. Guo, Integrated polarization rotator/converter by stimulated Raman adiabatic passage, Opt. Express 21, 17097 (2013).

[30] A. Espinosa-Soria and A. Martínez, Transverse spin and spinorbit coupling in silicon waveguides, IEEE Photon. Technol. Lett. 28, 1561 (2016).

[31] F. J. Rodríguez-Fortuño, I. Barber-Sanz, D. Puerto, A. Griol, and A. Martínez, Resolving light handedness with an on-chip silicon microdisk, ACS Photon. 1, 762 (2014).

[32] J. E. Vázquez-Lozano and A. Martínez, Towards chiral sensing and spectroscopy enabled by all-dielectric integrated photonic waveguides, arXiv:1911.11106 [physics.optics].

[33] D. Zhang, X. Feng, K. Cui, F. Liu, and Y. Huang, Generating in-plane optical orbital angular momentum beams with silicon waveguides, IEEE Photon. J. 5, 2201206 (2013). 
[34] S. Zheng and J. Wang, On-chip orbital angular momentum modes generator and (de)multiplexer based on trench silicon waveguides, Opt. Express 25, 18492 (2017).

[35] V. S. Lyubopytov, R. V. Kutluyarov, V. K. Bagmanov, N. Neumann, and A. K. Sultanov, Modeling and optimization of vortex modes propagation in rectangular dielectric waveguides, IEEE Photon. J. 12, 1 (2020).

[36] Y. Liang, H. W. Wu, B. J. Huang, and X. G. Huang, Light beams with selective angular momentum generated by hybrid plasmonic waveguides, Nanoscale 6, 12360 (2014).

[37] M. Onoda, S. Murakami, and N. Nagaosa, Hall Effect of Light, Phys. Rev. Lett. 93, 083901 (2004).

[38] K. Y. Bliokh, A. Y. Bekshaev, and F. Nori, Extraordinary momentum and spin in evanescent waves, Nat. Commun. 5, 3300 (2014).

[39] A. Aiello, P. Banzer, M. Neugebauer, and G. Leuchs, From transverse angular momentum to photonic wheels, Nat. Photon. 9, 789 (2015).

[40] D. Bhatti, J. von Zanthier, and G. S. Agarwal, Entanglement of polarization and orbital angular momentum, Phys. Rev. A 91, 062303 (2015).

[41] S. Longhi, Quantum-optical analogies using photonic structures, Laser Photon. Rev. 3, 243 (2009).

[42] O. Hosten and P. Kwiat, Observation of the spin Hall effect of light via weak measurements, Science 319, 787 (2008).
[43] K. Y. Bliokh, A. Y. Bekshaev, and F. Nori, Optical Momentum, Spin, and Angular Momentum in Dispersive Media, Phys. Rev. Lett. 119, 073901 (2017).

[44] J. D. Jackson, Classical Electrodynamics, 3rd ed. (Wiley, New York, 1999).

[45] A. T. O'Neil, I. MacVicar, L. Allen, and M. J. Padgett, Intrinsic and Extrinsic Nature of the Orbital Angular Momentum of a Light Beam, Phys. Rev. Lett. 88, 053601 (2002).

[46] A. Niv, G. Biener, V. Kleiner, and E. Hasman, Manipulation of the Pancharatnam phase in vectorial vortices, Opt. Express 14, 4208 (2006).

[47] D. Zhang, X. Feng, K. Cui, F. Liu, and Y. Huang, Identifying orbital angular momentum of vectorial vortices with Pancharatnam phase and Stokes parameters, Sci. Rep. 5, 11982 (2015).

[48] D. Zhang, X. Feng, and Y. Huang, Orbital angular momentum induced by nonabsorbing optical elements through space-variant polarization-state manipulations, Phys. Rev. A 98, 043845 (2018).

[49] F. Tamburini, B. Thidé, and M. Della Valle, Measurement of the spin of the M87 black hole from its observed twisted light, Mon. Not. R. Astron. Soc. Lett. 492, L22 (2020).

[50] H. A. Haus, Waves and Fields in Optoelectronics (Prentice-Hall, Upper Saddle River, NJ, 1984).

[51] A. B. Fallahkhair, K. S. Li, and T. E. Murphy, Vector finite difference modesolver for anisotropic dielectric waveguides, J. Lightwave Technol. 26, 1423 (2008). 